\title{
Globe
}

Revue internationale d'études québécoises

Sylvain Schryburt, De l'acteur vedette au théâtre de festival :

histoire des pratiques scéniques montréalaises 1940-1980, Montréal, Presses de l’Université de Montréal, 2011

\section{Hervé Guay}

Volume 15, numéro 1-2, 2012

URI : https://id.erudit.org/iderudit/1014643ar

DOI : https://doi.org/10.7202/1014643ar

Aller au sommaire du numéro

Éditeur(s)

Globe, Revue internationale d'études québécoises

ISSN

1481-5869 (imprimé)

1923-8231 (numérique)

Découvrir la revue

Citer ce compte rendu

Guay, H. (2012). Compte rendu de [Sylvain Schryburt, De l'acteur vedette au théâtre de festival : histoire des pratiques scéniques montréalaises 1940-1980, Montréal, Presses de l'Université de Montréal, 2011]. Globe, 15(1-2), 352-354. https://doi.org/10.7202/1014643ar d'utilisation que vous pouvez consulter en ligne.

https://apropos.erudit.org/fr/usagers/politique-dutilisation/ 


\section{Sylvain Schryburt}

De l'acteur vedette au théâtre de festival:

histoire des pratiques scéniques montréalaises 1940-1980,

Montréal, Presses de l'Université de Montréal, 2011.

Le nouvel ouvrage de Sylvain Schryburt sera un précieux outil pour aider le professeur de théâtre de niveau collégial et universitaire à préparer ses cours, car l'auteur produit avec ce livre une première synthèse des pratiques scéniques montréalaises des années 1940 jusqu’à 1980. D’un côté, «l'année 1980 marque la fin à la fois brutale et rapide du mouvement des collectifs de création des années 1970 » (p. 12). De l'autre, il fait remonter les débuts de l'histoire de la mise en scène au Québec à l'aventure de L'Équipe de Pierre Dagenais, première troupe, selon lui, à allonger la période de répétitions dévolue à l'élaboration du spectacle. Dans la mesure où ces quatre décennies comptent parmi les plus cruciales du développement de notre théâtre professionnel, il est heureux qu'un chercheur, prenant appui sur les abondantes recherches qui existent et grâce à la compulsion de multiples documents non encore dépouillés, fasse ressortir les principaux changements institutionnels et esthétiques qui ont marqué les scènes montréalaises durant cette période.

Si ce découpage historique se tient et est bien justifié, je suis moins convaincu de la thèse que Schryburt défend et qui nous vaut les appellations employées dans son titre pour désigner le mode de production des années 1940 (régime de l'acteur vedette) ainsi que celui qui émergerait au seuil des années 1980 (régime du théâtre de festival). Pour qu'il y ait régime de l'acteur vedette, il faudrait minimalement que l'un de ces acteurs possède ses propres moyens de production ou jouisse d'un grand prestige, comme c'était le cas au temps de l'actor-manager. Au reste, les vraies vedettes du temps ne sont-elles pas les stars du cinéma français et américain ? Or le Théâtre Arcade, qui sert à Schryburt à asseoir sa désignation, a beau aligner quelques vedettes, il appartient à France-Film et la production semble plutôt définie par JosephAlexandre DeSève, le propriétaire de l'entreprise. En effet, à partir du moment où ce dernier installe une troupe à l'Arcade, il décide du type de théâtre qu'elle jouera, ainsi que cela se passait avant la Première Guerre mondiale. De même, suffit-il que des festivals internationaux naissent au Québec au milieu des années 1980 pour que l'ensemble de l'activité théâtrale soit infléchi de manière à se "raccorde $[\mathrm{r}]$ au circuit international de tournées " (p. 360) ? Il est clair, cependant, que le théâtre commercial survit dans les 
années 1940 à Montréal et que davantage de troupes québécoises tournent à partir des années 1980 - ainsi que l'a observé Schryburt - sans qu'on puisse pour autant attribuer ces phénomènes à un seul facteur structurant.

Cette lecture très verticale de la dynamique théâtrale montréalaise tient sans doute aux fondements théoriques sur lesquels repose cet essai, à savoir la théorie du champ de Bourdieu et l'horizon d'attente de Jauss, appuis que Schryburt ne prend pas la peine d'adapter à la situation nordaméricaine ni au bilinguisme de la métropole. Il est significatif à cet égard qu'il écarte d'emblée le théâtre montréalais d'expression anglaise sans le justifier. Cela explique aussi son intérêt pour les pratiques les plus légitimées - surtout au début de l'ouvrage - avant que ne s'assouplisse son point de vue après l'éclosion de la dramaturgie québécoise et de la création collective à la fin des années 1960, moment de l'écriture où l'auteur sent le besoin d'ajouter des références théoriques, celles de Nicole Fortin (Une littérature inventée) en particulier, pour cerner un phénomène étranger aux thèses bourdieusiennes. Cet ajustement indique que Schryburt est à la fois capable de se montrer rigoureux dans l'application de son cadre théorique et de faire preuve de souplesse dans l'analyse quand les événements le réclament.

De l'acteur vedette au théâtre de festival compte quatre grandes parties. Dans chacune d'entre elles, le professeur de l'Université d'Ottawa nous fait part à la fois des changements institutionnels et esthétiques qui ont lieu. C'est d'ailleurs dans la saisie de l'évolution esthétique ayant cours à chacune des quatre périodes qu'il distingue que ses analyses s'avèrent les plus fines et éclairantes. Il use des archives et de la critique journalistique avec beaucoup de doigté pour repérer, par exemple, l'inspiration sociale et parfois brechtienne des Apprentis Sorciers, la "veine artaudienne" des Saltimbanques et l'emploi des techniques de projections sur la scène dans les années 1960.

La première partie ("L'émergence du metteur en scène (19371952)»), bien qu'elle soit très fouillée, m’est apparue un peu plus faible que les autres. Moins dans ses constatations générales et sa description du travail de L'Équipe et de celui des Compagnons (alerte et mesuré) que dans la manière dont Schryburt situe cet intervalle relativement aux périodes précédentes. Y a-t-il vraiment une filiation Nouveautés-Stella-Arcade comme il l'affirme? Joue-t-on encore dans les années 1940 comme on jouait avant la Première Guerre mondiale, d'autant que certains des acteurs de l'Arcade ont fait du cinéma? Le spectateur se déplace-t-il vraiment davantage pour voir un acteur qu'une pièce en particulier quand la troupe ne change guère durant l'année? Cela ne me paraît pas prouvé, tout au moins pas aussi bien étayé 
que les modèles artistiques et le type de scénographie auxquels ont recours les principales troupes de l'époque.

Nettement plus solides s'avèrent les trois parties suivantes, peutêtre parce qu'il existe une documentation fiable et nombreuse. Ainsi, l'auteur expose avec une grande clarté l'ascension du Théâtre du Nouveau Monde, qui impose " $[u] n$ modèle de pureté » artistique caractérisé, selon lui, par "un répertoire à haute tenue littéraire » (p. 106), par un jeu de l'acteur "gracieux de port, d'un maintien harmonieux " (p. 116) et par des "mise[s] en scène transparente $[\mathrm{s}]$ »(p. 116). Dans le chapitre suivant, intitulé «Les pressions de la marge, 1958-1969», l'effet « des multiples nouveautés, tant scéniques que dramaturgiques" (p. 129), introduites par les «théâtres de poche » dans le "champ montréalais" est fort bien décrit. Il en va de même de la troisième partie, plus longue, où sont observés tant les changements qui frappent le thêâtre institutionnel que le "régime collectif " privilégié par le Jeune Théâtre, à quoi s'ajoute le mode de fonctionnement des compagnies de recherche. Les productions du Théâtre expérimental de Montréal (1976-1979) sont notamment scrutées en détail et ces analyses comptent parmi les meilleures de l'ouvrage.

Le récit de ces quarante années déterminantes est enlevant dans la mesure où, le plus souvent, il synthétise ce qu'on savait du travail des multiples compagnies auxquelles il s'attarde, tout en montrant bien ce qui les distingue les unes des autres, particulièrement sur le plan esthétique. Les influences extérieures (Copeau, le Cartel, Brecht, Artaud, le Living Theatre, Grotowski, Barba, Decroux, etc.) sont indiquées, de même que sont signalés les écarts que les praticiens prennent avec leurs maitres. Bref, cette Histoire des pratiques scéniques montréalaises porte bien son nom et, en 400 pages, offre un condensé, sans trop de raccourcis et avec suffisamment de conceptualisation, d'un théâtre qui, en peu de temps, passe d'une activité purement commerciale à une institution en grande partie subventionnée par les pouvoirs publics.

Reste la thèse du régime de théâtre de festival sur laquelle se conclut cet essai. Je sais que Sylvain Schryburt y travaille et j'ose espérer qu'elle débouchera sur une meilleure compréhension de la dynamique institutionnelle et esthétique du théâtre québécois postérieur à la limite temporelle de son ouvrage. Car s'il est possible de remettre en question certaines des hypothèses d'un chercheur, il est encore plus stimulant de le voir en proposer dans le cadre d'un travail de grande ampleur.

Hervé Guay

Université du Québec à Trois-Rivières 